\title{
RF Driven 5G System Design for Centimeter Waves
}

\author{
Pekka Pirinen (D), Harri Pennanen, Ari Pouttu, Tommi Tuovinen, Nuutti Tervo, \\ Petri Luoto, Antti Roivainen, Aarno Pärssinen, and Matti Latva-aho
}

Centre for Wireless Communications, P.O. Box 4500, FI-90014 University of Oulu, Finland

Correspondence should be addressed to Pekka Pirinen; pekka.pirinen@oulu.fi

Received 22 December 2017; Accepted 12 April 2018; Published 23 May 2018

Academic Editor: Shao-Yu Lien

Copyright (c) 2018 Pekka Pirinen et al. This is an open access article distributed under the Creative Commons Attribution License, which permits unrestricted use, distribution, and reproduction in any medium, provided the original work is properly cited.

\begin{abstract}
5G system design is a complex process due to a great variety of applications and their diverse requirements. This article describes our experiences in developing a centimeter waves mobile broadband concept satisfying future capacity requirements. The first step in the process was the radio channel measurement campaign and statistical modeling. Then the link level design was performed tightly together with the radio frequency (RF) implementation requirements to allow as large scalability of the air interface as possible. We started the concept development at $10 \mathrm{GHz}$ frequency band and during the project World Radiocommunication Conference 2015 selected somewhat higher frequencies as new candidates for $5 \mathrm{G}$. Thus, the main learning was to gain insight of interdependencies of different phenomena and find feasible combinations of techniques and parameter combinations that might actually work in practice, not only in theory.
\end{abstract}

\section{Introduction}

All mobile communication system generations have had a clear key application driver: $1 G$ for analog voice, $2 G$ for digital voice and text messaging, $3 \mathrm{G}$ for multimedia and Internet connectivity, and $4 \mathrm{G}$ for true mobile broadband [1]. 5G needs to cooperate and internetwork seamlessly with these legacy networks as long as they are in operation and provide added value to the ecosystem. Foreseen application areas for $5 \mathrm{G}$ are much broader than in earlier generations, and therefore, such qualities as system parameter adaptation, scalability, reconfigurability, virtualization, and self-organization become necessities for $5 \mathrm{G}$.

This article overviews our approach to tackle the challenge of moving to higher center frequencies and address its implications to 5G system concept design. The starting point was to evaluate the new candidate frequencies in the centimeter wave bands that were investigated in METIS project [2-5]. Our selection at that point was $10 \mathrm{GHz}$ as it was the lowest frequency and thereby it would be less risky and more predictable to go there than jump to much higher uncharted frequencies. Then we planned and completed some measurement campaigns to evaluate the propagation characteristics at $10 \mathrm{GHz}$. Also, to keep the concept development manageable with limited resources, we decided to have the main emphasis on the flexible and scalable enhanced mobile broadband communications air interface with a well-established orthogonal frequency-division multiplexing (OFDM) waveform. An integral part of the work has been a tight linkage to RF implementation issues and crosschecking the feasibility of different communications options simultaneously from the RF perspective. This kind of comprehensive view on the practical system concept development is far too often ignored and is therefore highlighted in this article.

Now, being aware of the World Radiocommunication Conference (WRC) 2015 decisions that the lowest beyond 6 $\mathrm{GHz}$ serious candidate frequencies have moved to above 24 $\mathrm{GHz}$, the interest toward $10 \mathrm{GHz}$ band is fading. However, irrespective of the actual center frequency many of the lessons learned are common and transferrable to any concept development process when utilizing previously unexplored spectral resources and planning a new ultra-scalable communications platform.

Our main contributions in this paper are as follows:

(i) Set performance targets and key metrics for $5 \mathrm{G}$ system design. 
(ii) Provide high-level functional architecture of 5G network.

(iii) Present large-scale parameters derived from performed $10 \mathrm{GHz}$ channel measurements.

(iv) Define flexible physical layer parameterization, signaling, and multiaccess structure.

(v) Elaborate RF link budget, beamforming, and practical implementation issues that are critical to $5 \mathrm{G}$ system design and performance at centimeter waves.

The rest of the article discusses first various $5 \mathrm{G}$ use cases and their respective system design objectives. Then, 5G system concept design is described from the network architecture viewpoint. The next two sections consider channel measurements and modeling issues and link level design issues. Implications of merging RF architecture design and considerations to the concept are presented next. Finally, some concluding remarks are given.

\section{Use Cases and System Design Targets}

A fully evolved 5G system needs to support diverse application areas such as enhanced mobile broadband (eMBB), massive Internet of things (MIoT), and mission-critical communications (MCC) [1]. All these use cases have distinct and partly contradictory requirements in terms of their key performance indicators, making the system concept design, as a whole, extremely complex. In most of the cases, not all of the requirements need to be simultaneously met. Thus, advanced 5G infrastructures move away from a "one architecture fits all" nature towards a "multiple architectures adapted to each service" concept. In this paper, the $5 \mathrm{G}$ system concept is mainly designed for eMBB, whereas the equally important use cases of MIoT and MCC have earned a fair share of attention as exemplified in $[2,6,7]$. In addition to extremely high throughputs, another main aspect of eMBB is the total system capacity. The ultra-high density of broadband user connections needs to be supported as well. New spectrum allocations, cell densification, and massive MIMO technology are seen as the key enablers to achieve these challenging goals.

The key design targets of the proposed 5G concept are presented below. These targets are the theoretical maximums that the system could support in ideal conditions.

(1) Support for scalable bandwidths up to $0.5-1 \mathrm{GHz}$ in carrier frequencies around 10 and $30 \mathrm{GHz}$.

(2) Peak data rate that scales with system bandwidth, meaning tens of Gbps for $0.5-1 \mathrm{GHz}$ bandwidths.

(3) Supported antenna and stream configurations:

(a) Max. 256 transmit (TX) antennas and 16 receive (RX) antennas.

(b) Max. 16 independent data streams.

(4) Spectrum efficiency

(a) Max. 100 bits/s/Hz.

(5) Latency: (a) Control plane: $<10 \mathrm{~ms}$ to establish user plane.

(b) User plane: $<1 \mathrm{~ms}$ from the user to server.

(6) Mobility:

(a) Home and office, optimized for speeds $<5 \mathrm{~km} / \mathrm{h}$

(b) Extreme mobility, speeds up to $500 \mathrm{~km} / \mathrm{h}$.

(7) Coverage:

(a) Indoor coverage up to $30 \mathrm{~m}$.

(b) Outdoor coverage up to $300 \mathrm{~m}$.

(c) Operation $>300 \mathrm{~m}$ using lower $\mathrm{cm}$-wave frequencies.

Theoretical values for maximum data rates are calculated with respect to the allocated spectrum in Section 5. The maximum multiple-input multiple-output (MIMO) configuration set for $5 \mathrm{G}$ system concept is $256 \times 16$ with the maximum number of 16 data streams. If this MIMO deployment can be achieved, the maximum spectrum efficiency level may be up to $100 \mathrm{bit} / \mathrm{s} / \mathrm{Hz}$ given that 256QAM (quadrature amplitude modulation) is used. 256QAM requires error vector magnitude (EVM) levels of $-33 \mathrm{~dB}$, which sets rather stringent design targets for RF chain designs. The RF architecture aspects of 5G system concept are discussed in Section 6. One key target of $5 \mathrm{G}$ system is to define signaling structures, which enable very low delays in data transmission. The $5 \mathrm{G}$ systems should be able to provide $10 \mathrm{~ms}$ end-to-end (E2E) latency in general and $1 \mathrm{~ms}$ in extremely low latency use cases. The stringent latency requirements are addressed in the link level physical resource block design.

\section{Network Architecture}

The main target is to find critical solutions for a system concept suitable for a small cell deployment scenario of $5 \mathrm{G}$. Furthermore, the design of $5 \mathrm{G}$ network architecture should be flexible. The current thinking is that there are such public spaces as stadiums and city centers wherein existing network operators shall have a role for deploying the access points and operating the networks. On the other hand, there are privately owned properties (housing blocks and shopping malls) where the network access points are privately/corporate owned or rented and the deployment and operation may be purchased from a new type of network operator. The following assumptions are used in the network level system design:

(i) Infrastructure sharing between operators allowed

(ii) Small cells and dedicated spectrum

(iii) Multioperator environment

(iv) Private networks, private access points

(v) Support for contention-based and scheduled resource usage

The generic functional architecture model for $5 \mathrm{G}$ system concept illustrating the basic functional entities of the control 
and user plane in the device and network infrastructure parts is shown in Figure 1. The physical location of the functional entities in the infrastructure part can vary between radio nodes and more centralized units depending on the practical network deployment. The small cell radio networks are connected to common core cloud network ("EPC", Evolved Packet Core type network) with high capacity connections. Common core cloud network can serve multiple operators. The connectivity management related functionalities (for example, mobility management) are implemented in the core cloud network. The radio access network related functionalities could be distributed between the local radio network and the common core cloud network. The location of different functionalities, such as radio resource control and air interface management, may depend on the local RAN, core cloud network connection quality. In a general case, we can assume that air interface L1/L2 control is placed close to access points, while higher layers could be centrally located into the core cloud network. If very high speed connections (fiber cable) are available, then also the air interface L1/L2 control could be implemented into servers located in the core cloud network.

\section{Radio Channel Models for System Design}

Appropriate channel model is a starting point and mandatory for any system design. In the geometry-based stochastic channel model (GSCM), the propagation channel is characterized by statistical parameters obtained from the radio channel measurements. This gives a possibility of using the same framework of the model for the simulations in different frequencies and the different number or type of antennas. Due to missing characterizations of propagation channel at $10 \mathrm{GHz}$ and in order to utilize GSCM promptly at $10 \mathrm{GHz}$ frequency band, we carried out radio channel measurement campaigns with vector network analyzer and virtual arrays in the campus area of the University of Oulu. The measurements covered two different propagation scenarios, namely, twostory lobby and urban small cell scenarios. From the collected measurement data, complete parameterizations were derived for three-dimensional (3D) GCSM. The parameterizations are directly applicable to the 3rd Generation Partnership Project (3GPP) model [8].

The most important large-scale (LS) characteristics of the propagation channel are path loss and shadow fading. Based on our results, the path loss models are in some extent similar than in other frequency bands. However, the standard deviation of shadow fading $\sigma_{\mathrm{SF}}$ is significantly smaller due to static measured propagation environment. In addition to the path loss models and $\sigma_{\mathrm{SF}}$, the parameterizations consisted of 50 different propagation parameters. For example, LS parameters are modeled by log-normal distribution with specific mean $\mu$ and standard deviation $\sigma$ values giving higher level characterization of the propagation channel. Determined LS parameters are summarized in Table 1 and the full set of parameters is presented in $[9,10]$.

Although the model parameters are heavily dependent on the measured propagation environment, the following conclusions can be drawn from the determined LS and smallscale parameters: (i) Parameters describing the delay and angular dispersion, that is, DS and angle spreads (ASs), seem to decrease in comparison to the parameters in the existing models at the frequency bands below $6 \mathrm{GHz}$ due to the higher attenuation of delayed components.

(ii) When compared with lower frequency bands, specular reflection is more dominant propagation mechanism in comparison to diffuse scattering, leading to smaller cluster ASs.

Also, several research projects including industry and academia have been targeting to fulfill the requirements for designing and evaluating new channel models for the frequency bands up to $100 \mathrm{GHz}$. For instance, the initial parameterizations have been proposed for extending the quasi-deterministic radio channel generator (QuaDRiGa) over 10-80 GHz frequency band in [11]. Also, METIS project [12] addressed the challenges of the future channel modeling and recently developed a new map-based channel model up to $86 \mathrm{GHz}$ as a pioneering work for $5 \mathrm{G}$ mobile communication system evaluations.

METIS model was intended to take into account all radio channel characteristics, which are important for any 5G mobile communications scenario. The model is based on the ray-tracing (RT) using a simplified $3 \mathrm{D}$ geometric description of the propagation environment. In the model, the building walls are modeled as rectangular surfaces with specific electromagnetic material properties, and the propagation paths are modeled deterministically. However, the model is not fully deterministic since the random objects representing for instance people and vehicles on the radio link are modeled stochastically. Therefore, the model can be understood as semideterministic model having significantly shorter processing time in comparison to traditional RT. Even though several properties of the model have already been successfully validated, the model still needs to be validated by additional measurements.

\section{Link Level Design}

5.1. Physical Layer Design. The physical layer design of the $5 \mathrm{G}$ system concept is based on the "OFDM signals with new numerology" approach. The signal structure has originally been designed to operate at $10 \mathrm{GHz}$ band with bandwidths up to $1 \mathrm{GHz}$. Key OFDM signal parameters are selected so that synergy remains with the existing Long Term Evolution (LTE) radio implementations. Also, we shall use the same channel coding solutions as with LTE, where appropriate. However, in general the backwards compatibility with LTE is not maintained, since the $5 \mathrm{G}$ requirements lead to different physical layer designs when aiming to optimize the system performance.

The subcarrier spacing has been selected as $120 \mathrm{kHz}$. With subcarrier spacing of $120 \mathrm{kHz}$, the useful symbol duration becomes $8.3 \mu \mathrm{s}$. The cyclic prefix should be short compared to the symbol duration, but long enough to eliminate impairments in the OFDM signal detection due to propagation channel. 


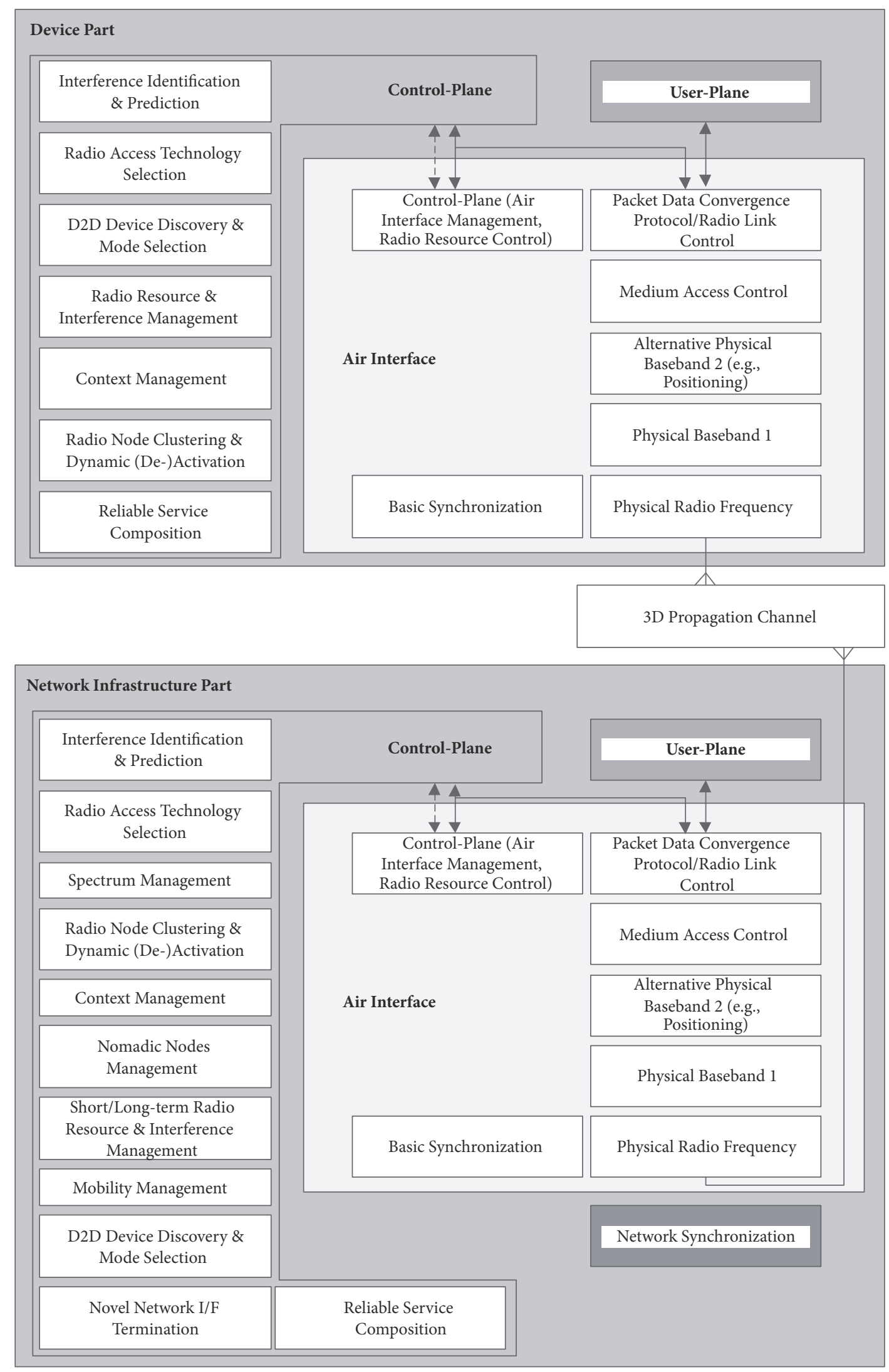

FIgURE 1: Functional network architecture. 


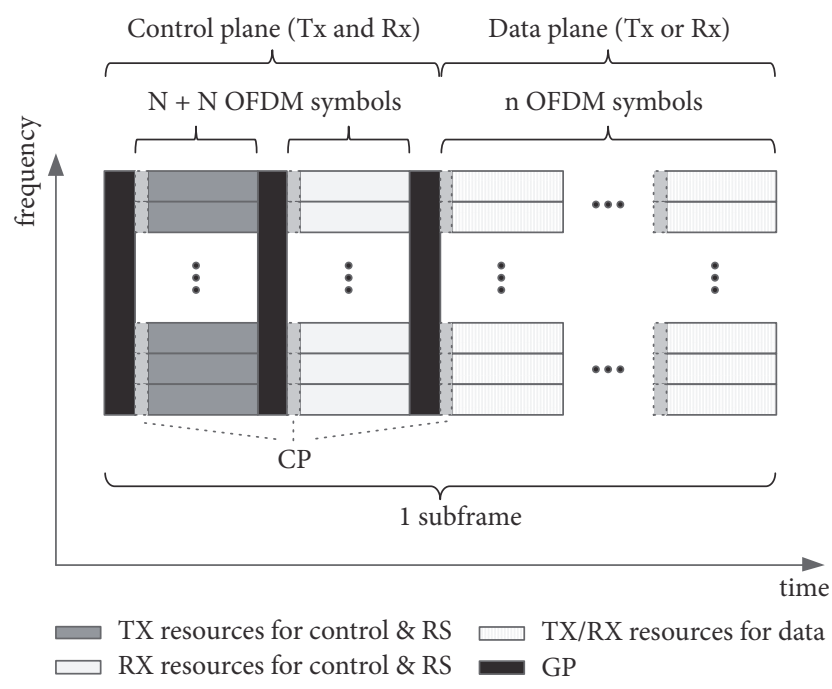

FIgure 2: Physical layer subframe structure.

For the small cells (cell radius in the order of $75 \mathrm{~m}$ ), cyclic prefix (CP) duration of $0.5 \mu$ s is large enough to prevent overlapping of OFDM symbols due to propagation delays even if no timing alignment is used in the UL transmissions. Measured delay spread values indicate that a typical delay spread at $10 \mathrm{GHz}$ frequency with short transmission distances in indoor and outdoor environments is below 50 ns. For these reasons, we conclude that $\mathrm{CP}$ duration of $0.5 \mu \mathrm{s}$ is enough for the $5 \mathrm{G}$ small cell operation scenarios. Table 2 shows alternative values for the $5 \mathrm{G}$ physical layer parameters, covering several (but not all possible) channel bandwidths between $31.25 \mathrm{MHz}$ and $1000 \mathrm{MHz}$.

Given the above physical layer design, we have also computed the achievable data rates for several parameter sets as indicated by Table 2 . The maximum data rates for a given bandwidth are achieved for 256QAM, 16 MIMO data streams, and coding rate $r=1$, while the minimum data rates are achieved for BPSK, 1 data stream, and coding rate $r=1 / 2$. We assume $90 \%$ bandwidth efficiency and rather optimistic protocol efficiency of $100 \%$.

As the duplexing method we have chosen asymmetric dynamic time division duplexing [13]. In this particular approach, the uplink and downlink capacities may be chosen based on the traffic need within each cell, interference mitigation, and/or management requirements and user densities.

5.2. Multiple Access Design. The $5 \mathrm{G}$ subframe structure is shown in Figure 2. One subframe contains 11 OFDM symbols in time domain. In the control plane ( $n=2$ symbols), we introduce a guard period of $\mathrm{T}_{\mathrm{GP}}=0.94 \mu \mathrm{s}$, and the cyclic prefix is $\mathrm{T}_{\mathrm{CP}}=0.5 \mu \mathrm{s}$. The OFDM symbol duration is $\mathrm{T}_{\text {symbol }}=$ $8.33 \mu \mathrm{s}$. Therefore, for the subframe duration, we get $\mathrm{T}_{\text {subframe }}$ $=3 \cdot \mathrm{T}_{\mathrm{GP}}+11 \cdot\left(\mathrm{T}_{\text {symbol }}+\mathrm{T}_{\mathrm{CP}}\right)=100 \mu \mathrm{s}$.

In the case where the system does not request extremely small E2E latencies (1 ms), the amount of control overhead can be reduced by concatenating multiple subframes together. In this case, the TX and RX control parts are embedded into the first subframe with 9 data symbols, while the remaining subframes (11 symbols) contain only data plane signal (either TX or RX) with first three symbols in each concatenated subframe having an extended cyclic prefix of $\mathrm{T}_{\mathrm{CP} \text {,EXT }}=$ $1.44 \mu \mathrm{s}$.

We propose that the system shall have random access (contention-based) and scheduled resources. For the scheduled resources, we shall use orthogonal frequency-division multiple access (OFDMA), where the resource block (RB) size is a compromise between high granularity (to support transmission of very low amount of data) and signaling overhead. The minimum RB size is selected here as 72 resource elements, consisting of 8 consecutive subcarriers and 9 data plane symbols (note: resource element is defined as 1 subcarrier and 1 data plane symbol). This is comparable to LTE resource block size (84 resource elements) and can work both with machine-type services and mobile broadband data.

Random access resources shall be used by simple IoT devices, which are constrained by small form factor and/or battery operation. The scheduled resources can be used by more complex IoT devices and especially mobile cellular users as well as mobile broadband customers. The sharing between the resources shall be handled by the spectrum manager described earlier.

In the contention-based medium access case, the Resource Coordination functionality of layered resource management provides the template frame to a cluster of nodes allowing contention-based access inside the cluster. Control signals are transmitted in time-frequency resources separated from the data resources.

\section{Towards 5G RF Implementation}

Large antenna arrays will be one of the key enablers for 5G RF implementations for both capacity and link range. In this section, we briefly discuss multibeam link budget and $\mathrm{RF}$ restrictions for implementing $\mathrm{cm}$-wave multiantenna transceivers (TRXs). Further in-depth discussion on the subject is available in [15].

6.1. Link Budget. For achieving the target data rates in practice, the link budget must address at least the following:

(i) Capacity evaluations with different modulations and waveforms

(ii) Hardware assumptions including physical dimensions, power, noise, and nonlinearity

(iii) Partitioning of signal-to-noise ratio (SNR) budget for different parts of TX and RX

(iv) Multistream transmission and adaptive beamforming

(v) Spatial channel model

Practical RF link budget consists of optimizing tens of different parameters together for setting the design targets for TRX design. Furthermore, requirements are very dependent on the target scenario including waveform assumptions, propagation environment, required physical dimensions, and user positions. Table 3 presents an example of system level RF specifications for two different frequency bands at indoor 
TABLE 1: Derived LS parameters from the channel measurements.

\begin{tabular}{|c|c|c|c|c|c|}
\hline \multirow{2}{*}{\multicolumn{2}{|c|}{ Channel model parameter }} & \multicolumn{2}{|c|}{ LOS } & \multicolumn{2}{|c|}{ NLOS } \\
\hline & & \multirow{2}{*}{$\begin{array}{c}\text { Two-story lobby } \\
-7.78\end{array}$} & \multirow{2}{*}{$\frac{\text { Urban small cell }}{-7.70}$} & \multirow{2}{*}{$\begin{array}{c}\text { Two-story lobby } \\
-7.55\end{array}$} & \multirow{2}{*}{$\frac{\text { Urban small cell }}{-7.41}$} \\
\hline DS $\log _{1}([s])$ & $\mu_{\mathrm{DS}}$ & & & & \\
\hline$D S \log _{10}([\mathrm{~S}])$ & $\sigma_{\mathrm{DS}}$ & 0.13 & 0.16 & 0.17 & 0.14 \\
\hline \multirow{2}{*}{$\mathrm{KF}[\mathrm{dB}]$} & $\mu_{\mathrm{KF}}$ & 8.5 & 5.1 & N/A & $\mathrm{N} / \mathrm{A}$ \\
\hline & $\sigma_{\mathrm{KF}}$ & 3.5 & 3.2 & N/A & N/A \\
\hline $\mathrm{SF}[\mathrm{dB}]$ & $\sigma_{\mathrm{SF}}$ & 2 & 2 & 3 & 2 \\
\hline \multirow{2}{*}{$\operatorname{ASD} \log _{10}\left(\left[^{\circ}\right]\right)$} & $\mu_{\mathrm{ASD}}$ & 0.86 & 1.08 & 1.32 & 1.24 \\
\hline & $\sigma_{\mathrm{ASD}}$ & 0.23 & 0.35 & 0.23 & 0.32 \\
\hline \multirow{2}{*}{ ASA $\log _{10}\left(\left[^{\circ}\right]\right)$} & $\mu_{\mathrm{ASA}}$ & 1.44 & 1.47 & 1.64 & 1.77 \\
\hline & $\sigma_{\mathrm{ASA}}$ & 0.11 & 0.20 & 0.18 & 0.08 \\
\hline \multirow{2}{*}{$\operatorname{ESD} \log _{10}\left(\left[^{\circ}\right]\right)$} & $\mu_{\mathrm{ESD}}$ & 0.91 & 0.80 & 0.54 & 0.89 \\
\hline & $\sigma_{\mathrm{ESD}}$ & 0.31 & 0.17 & 0.49 & 0.07 \\
\hline \multirow{2}{*}{ ESA $\log _{10}\left(\left[^{\circ}\right]\right)$} & $\mu_{\mathrm{ESA}}$ & 0.61 & 1.12 & 0.82 & 1.08 \\
\hline & $\sigma_{\mathrm{ESA}}$ & 0.17 & 0.10 & 0.29 & 0.13 \\
\hline
\end{tabular}

$\mathrm{DS}$ = root mean square delay spread; $\mathrm{KF}=$ Rician $\mathrm{K}$-factor; $\mathrm{SF}=$ shadow fading; $\mathrm{ASD}=$ azimuth angle spread of departure; ASA = azimuth angle spread of arrival; ESD = elevation angle spread of departure; and ESA = elevation angle spread of arrival.

TABLE 2: Physical layer signal parameters for $5 \mathrm{G}$ system concept (LTE values as a reference).

\begin{tabular}{|c|c|c|c|c|c|}
\hline Property & LTE & & & $10 \mathrm{G}$ & \\
\hline Channel BW [MHz] & 20 & 31.25 & 125 & 500 & 1000 \\
\hline Subframe length [ms] & 1 & 0.1 & 0.1 & 0.1 & 0.1 \\
\hline Sampling frequency $[\mathrm{MHz}]$ & 30.72 & 30.72 & 122.88 & 491.52 & 983.04 \\
\hline FFT size & 2048 & 256 & 1024 & 4096 & 8192 \\
\hline Subcarrier spacing $[\mathrm{kHz}]$ & 15 & 120 & 120 & 120 & 120 \\
\hline Occupied subcarriers & 1201 & 234 & 938 & 3750 & 7500 \\
\hline Guard subcarriers & 847 & 22 & 86 & 346 & 692 \\
\hline Occupied bandwidth [MHz] & 18.015 & 28 & 113 & 450 & 900 \\
\hline DL BW efficiency & $90 \%$ & $89.9 \%$ & $90 \%$ & $90 \%$ & $90 \%$ \\
\hline OFDM symbols/subframe & 7 & 11 & 11 & 11 & 11 \\
\hline Symbol duration excl. CP $[\mu \mathrm{s}]$ & 66.7 & 8.33 & 8.33 & 8.33 & 8.33 \\
\hline $\mathrm{CP}$ duration $[\mu \mathrm{s}]$ & $5.2 / 4.69$ & 0.5 & 0.5 & 0.5 & 0.5 \\
\hline Data rate on full BW Min ... Max [Mbps] & & $13 \ldots 3,391$ & $53 \ldots 13,592$ & $211 \ldots 54,340$ & $422 \ldots 108,680$ \\
\hline
\end{tabular}

LOS scenario. These very abstracted requirements must then be divided further for different parts of the TRX.

In practice, adaptive modulation and coding scheme defines the minimum SNR required at the RX input. The nonidealities of TX limit the achievable SNR with respect to absolute power level. Figure 3(a) shows an example of signal-to-noise plus distortion ratio (SNDR) simulated with OFDM/256QAM waveform and commercial linear power amplifier (PA) for $10 \mathrm{GHz}$. These results are then combined with EVM values of other parts of the TX. It is clearly observed that the achievable linear power and hence data rate is easily overestimated, if only some of the TX nonidealities are taken into account. RX is treated in a similar fashion. Furthermore, the overall SNR-budget must be distributed between TX and RX. In RX, the SNR is typically limited by the noise at lower signal levels, whereas other nonidealities including phase noise of the synthesizer, analog-to-digital converter (ADC) quantization noise, I/Q mismatches, and cochannel interference limit the SNR at higher power levels.
In MIMO systems, spatial channel model is required for link budget evaluations. Multistream link budget is determined based on MIMO beam-specific path gains, where each stream is handled as an independent link [16]. The required TX power for rank 1-4 data transmissions at $10.1 \mathrm{GHz}$ in indoor LOS scenario is presented in Figure 3(b). The used TX configuration is given in Table 3 including the notations: base station (BS), uniform rectangular array (URA), mobile terminal (MT), half-power beamwidth (HPBW), uniform linear array (ULA), and output power (Pout).

6.2. Beamforming Arrays for 5G. One of the fundamental questions in $\mathrm{cm}$-wave communications is the number of antennas. The direct consequence of increasing frequency is the decreased antenna aperture. Hence, we can increase the number of antennas while maintaining the same physical area and eventually provide more beamforming gain. Multiple antennas are not only needed for increasing the data rate, but fundamentally for providing any reasonable link range. High 
TABLE 3: Example of system level RF specifications.

\begin{tabular}{lcc}
\hline Center frequency & $\mathbf{1 0 . 1 ~ G H z}$ & $\mathbf{2 6 ~ G H z}$ \\
\hline Signal bandwidth & $500 \mathrm{MHz}$ & $1000 \mathrm{MHz}$ \\
Array configuration at BS & $8 \times 2 \mathrm{URA}$ & $16 \times 4 \mathrm{URA}$ \\
BS HPBW (azim, elev) & $\left(15^{\circ}, 90^{\circ}\right)$ & $\left(7^{\circ}, 30^{\circ}\right)$ \\
Array configuration at MT & 4 -element ULA & 8 -element ULA \\
MT HPBW (azim, elev) & $\left(30^{\circ}, 120^{\circ}\right)$ & $\left(7^{\circ}, 120^{\circ}\right)$ \\
TX EVM for uncoded 256 QAM & $2.2 \%$ & $2.2 \%$ \\
PA back-off (for OFDM signal) & $9.6 \mathrm{~dB}$ & $9.6 \mathrm{~dB}$ \\
Pout Peak per PA in (BS, MT) & $(10,0.1) \mathrm{W}$ & $(1,0.01) \mathrm{W}$ \\
Total Noise Figure of (BS, MT) & $(8,10) \mathrm{dB}$ & $(8,10) \mathrm{dB}$ \\
\hline
\end{tabular}

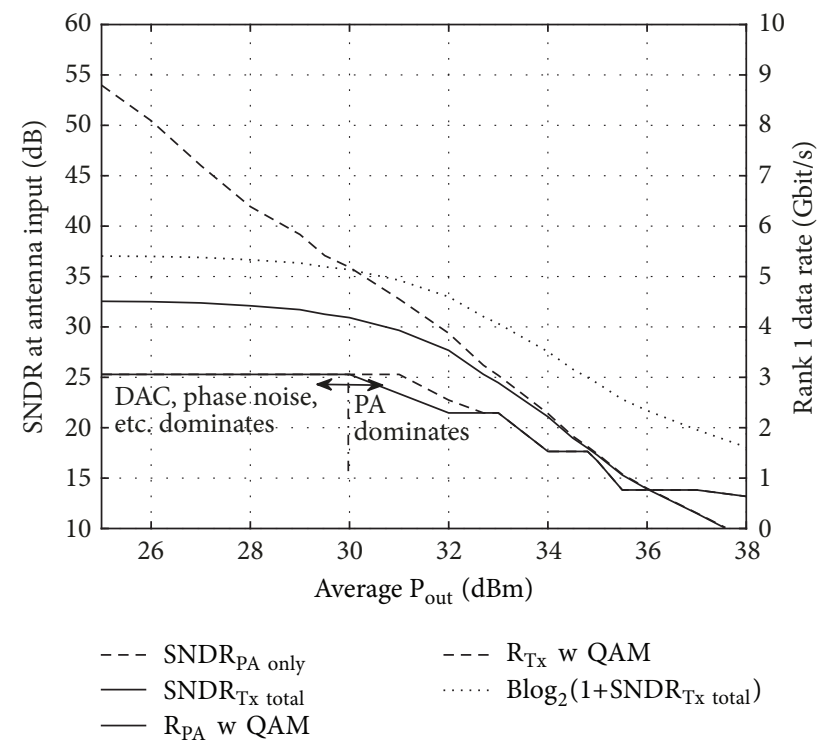

(a)

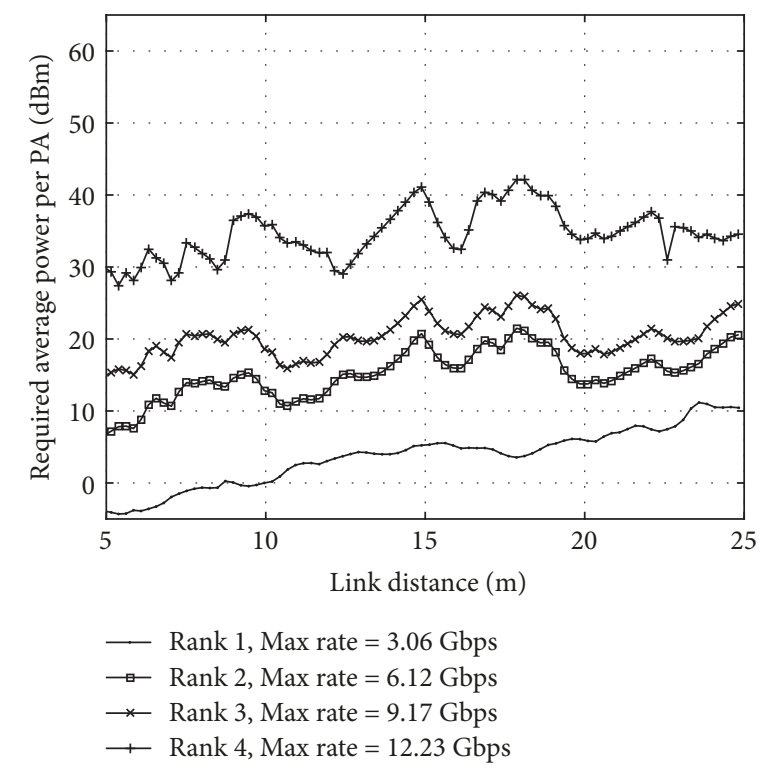

(b)

FIgURE 3: (a) Nonlinear PA and TX SNDR models with achievable data rates and (b) required average powers per PA for Rank N transmission with indoor LOS channel model [14] at $10.1 \mathrm{GHz}$.

directivity of the array results easily in very high effective isotropic radiated power (EIRP), which might be harmful for human tissue in case it is close to human body, limiting the maximum EIRP to be used. Decreasing antenna aperture also enables arrays for mobile terminals. However, the area of feeding network for large arrays limits the size of the antenna array in small form factors. Furthermore, because of higher circuit-level losses, the RF frontend must be embedded close to antennas to maintain the power efficiency.

In order to achieve the benefits of multiple antennas, beamforming system must be controlled adaptively. Traditionally, each antenna has individual RF chain and the control can be done in digital domain. However, because of extremely wide bandwidths, the TRX power consumption is not dominated only by analog components such as PA. Moreover, digital parallelism and wideband ADC's/digital-to-analog converters (DACs) are playing a crucial role when minimizing the power consumption. Because of these aspects, hybrid/RF beamforming is considered as de facto in $\mathrm{cm}$-wave cellular systems. Adaptive RF phase and amplitude control of individual antenna elements is essential for maintaining the connection in adaptive user scenarios and minimizing the interference between data transmissions. However, the array scanning has an impact on the impedance matching of individual elements. Furthermore, the single element pattern affects the array scanning region. Hence, practical assumption of array scanning angle is in range of $+/-30^{\circ}$.

6.3. Practical Design Challenges. The key challenges in $\mathrm{cm}-$ wave RF design are as follows:

(i) Wideband ADC/DAC dynamic range versus power consumption

(ii) Synthesizer phase noise

(iii) Linear (enough) output power with $\mathrm{cm}$-wave PAs

(iv) Implementing high-efficiency PA array

(v) Physical form factors in antenna-RF integration

(vi) RF- and hybrid-beamforming array design. 
The required sum power will be produced with several PA elements, resulting in decreased power per PA. Furthermore, multiple signals with different power levels in PA input give practical constraints for beam synthesis and power allocation per PA. These aspects give new tradeoffs for PA technologies although producing power at any of the options from CMOS to $\mathrm{GaN}$ will be a major challenge at high frequencies. The practical PA solution must be cheap, power efficient, linear, and small. However, these requirements cannot be optimized independently. Power efficient PA architectures, such as Doherty [17], are physically larger, cost more, and require linearization, which is traditionally done by digital predistortion (DPD). For RF/hybrid-beamforming arrays, conventional DPD is not possible because the waveform at each PA input cannot be controlled.

Different use scenarios set very different requirements for the RF implementation, especially in terms of required power, linearity, and beamforming. From the array scanning perspective, a convenient location of indoor BS is in the corner of the room/office area. A typical room layout indicates that it might be beneficial to have wider beams in the elevation than in the azimuth domain for beam/user separation. For outdoor BSs, high array gain for cell-edge users is a necessity that results in narrow beams. This complicates the beam scanning and tracking when serving mobile users. On the other hand, benefits of spatial filtering in network level management become apparent.

As the BS array design can be site-specific, the MT must adapt to various propagation scenarios. At $10 \mathrm{GHz}$, the practical number of elements varies between 2 and 8 with $\lambda / 2$ antenna spacing in small devices. Hence, a linear array is considered to be the only practical solution, because the impact of mechanics forces the array to be designed at the bottom or top end of a device. However, other device types such as tablets and laptops may contain more antennas.

\section{Conclusions and Way Forward}

Various 5G system concept design aspects at centimeter waves were discussed. As the main use case, we selected eMBB built upon OFDM and set the key performance targets for it. One of the first objectives was to measure and characterize the radio channel in the chosen new $\mathrm{cm}$-frequency. Based on the channel characteristics, link level design was carried out. It provides great adaptivity in air interface parameter setting, access schemes, and duplexing. Feasibility of the design objectives was checked in parallel with RF implementation aspects so that, for example, multiantenna beamforming, link budget, and power efficiency were be taken as integral system design elements. It was concluded that the high data rates achieved by high-order modulations and MIMO set extreme challenges for the nonlinearity, noise, and physical form factors of the RF devices. Based on this study, it is clear that RF design should be always the key driver when designing $5 \mathrm{G}$ solutions for centimeter waves and above that.

Millimeter-wave communications [18-22] are gaining increasing interest in $5 \mathrm{G}$ as the largest chunks of spectra are available at the bands above $30 \mathrm{GHz}$. Due to broader contiguous bands the link level design can be somewhat relaxed and yet very high data rates are achievable. Propagation and penetration losses tend to increase with frequency, limiting the feasible link range. However, at the same time antenna size and spacing go down enabling easier deployment of large MIMO systems with high array gain and beam directivity. Therefore, hybrid-beamforming architectures and powerand cost-efficient RF transceiver design remain in the focal role when moving from centimeter waves to millimeterwaves.

\section{Conflicts of Interest}

The authors declare that there are no conflicts of interest regarding the publication of this paper.

\section{Acknowledgments}

This article is mostly based on the research work in the "5G Radio Access Solutions $10 \mathrm{GHz}$ and Beyond Frequency Bands (5Gto10G)" project over the years 2014-2017. Project partners Bittium, Huawei, Keysight, Nokia, and Tekes are hereby gratefully acknowledged for their support.

\section{References}

[1] Qualcomm. Technologies, "Inc., Making 5G NR a reality Leading the technology innovations for a unified, more capable $5 \mathrm{G}$ air interface," White Paper, 2016.

[2] Mobile., "Mobile and Wireless Communication Enablers for the Twenty-twenty Information Society (METIS)," in FP7-ICT317669 Project, https://www.metis2020.com.

[3] A. Osseiran, F. Boccardi, V. Braun et al., "Scenarios for 5G mobile and wireless communications: the vision of the METIS project," IEEE Communications Magazine, vol. 52, no. 5, pp. 2635, 2014.

[4] H. Tullberg, P. Popovski, Z. Li et al., "The METIS 5G System Concept: Meeting the 5G Requirements," IEEE Communications Magazine, vol. 54, no. 12, pp. 132-139, 2016.

[5] J. F. Monserrat, G. Mange, V. Braun, H. Tullberg, G. Zimmermann, and Ö. Bulakci, "METIS research advances towards the 5 G mobile and wireless system definition," EURASIP Journal on Wireless Communications and Networking, vol. 2015, no. 1, pp. 1-16, 2015.

[6] FANTASTIC-5G, “5GPPP Project,” http://fantastic5g.com.

[7] C. Bockelmann, N. Pratas, H. Nikopour et al., "Massive machine-type communications in 5g: Physical and MAC-layer solutions," IEEE Communications Magazine, vol. 54, no. 9, pp. 59-65, 2016.

[8] “a 3rd Generation Partnership Project (3GPP)," Study on channel model for frequency spectrum above $6 \mathrm{GHz}$ TR 38.900 v14.0.0", 2016.

[9] A. Roivainen, C. Ferreira Dias, N. Tervo, V. Hovinen, M. Sonkki, and M. Latva-aho, "Geometry-based stochastic channel model for two-story lobby environment at $10 \mathrm{GHz}$," Institute of Electrical and Electronics Engineers. Transactions on Antennas and Propagation, vol. 64, no. 9, pp. 3990-4003, 2016.

[10] A. Roivainen, P. Kyösti, C. F. Dias et al., "Parametrization and validation of geometry-based stochastic channel model for urban small cells at $10 \mathrm{GHz}$," IEEE Transactions on Antennas and Propagation, vol. 65, no. 7, pp. 3809-3814, 2017. 
[11] "H2020-ICT-671650 Millimetre Wave Based Mobile Radio Access Network for Fifth Generation Integrated Communications (mmMAGIC) project," Measurement campaigns and initial channel models for preferred suitable frequency ranges Deliverable D2.1, 2016.

[12] H. Shokri-Ghadikolaei, C. Fischione, G. Fodor, P. Popovski, and M. Zorzi, "Millimeter Wave Cellular Networks: A MAC Layer Perspective," IEEE Transactions on Communications, vol. 63, no. 10, pp. 3437-3458, 2015.

[13] P. Jayasinghe, A. Tolli, and M. Latva-aho, "Bi-directional signaling strategies for dynamic TDD networks," in Proceedings of the 2015 IEEE 16th International Workshop on Signal Processing Advances in Wireless Communications (SPAWC), pp. 540-544, Stockholm, Sweden, June 2015.

[14] T. Tuovinen, N. Tervo, and A. Parssinen, "RF system requirement analysis and simulation methods towards $5 \mathrm{G}$ radios using massive MIMO," in Proceedings of the 2016 46th European Microwave Conference (EuMC), pp. 142-45, London, United Kingdom, October 2016.

[15] T. Tuovinen, N. Tervo, and A. Parssinen, "Analyzing 5G RF System Performance and Relation to Link Budget for Directive MIMO," IEEE Transactions on Antennas and Propagation, vol. 65, no. 12, pp. 6636-6645, 2017.

[16] T. Tuovinen, N. Tervo, and A. Pärssinen, "Downlink Output Power Requirements with an Experimental-Based Indoor LOS/ NLOS MIMO Channel Models at $10 \mathrm{GHz}$ to Provide $10 \mathrm{Gbit} / \mathrm{s}$," in Proceedings of the 46th European Microwave Conference, EuMC 2016, pp. 505-508, gbr, October 2016.

[17] R. S. Pengelly, “The Doherty power amplifier," in Proceedings of the 2015 IEEE MTT-S International Microwave Symposium (IMS2015), pp. 1-4, Phoenix, AZ, USA, May 2015.

[18] T. Rappaport, S. Sun, R. Mayzus et al., "Millimeter wave mobile communications for $5 \mathrm{G}$ cellular: it will work!," IEEE Access, vol. 1, pp. 335-349, 2013.

[19] S. Rangan, T. S. Rappaport, and E. Erkip, "Millimeter-wave cellular wireless networks: potentials and challenges," Proceedings of the IEEE, vol. 102, no. 3, pp. 366-385, 2014.

[20] R. W. Heath, N. Gonzalez-Prelcic, S. Rangan, W. Roh, and A. M. Sayeed, "An overview of signal processing techniques for millimeter wave MIMO systems," IEEE Journal of Selected Topics in Signal Processing, vol. 10, no. 3, pp. 436-453, 2016.

[21] M. Xiao, S. Mumtaz, Y. Huang et al., "Millimeter Wave Communications for Future Mobile Networks (Guest Editorial), Part I," IEEE Journal on Selected Areas in Communications, vol. 35, no. 7, pp. 1425-1431, 2017.

[22] L. Li, D. Wang, X. Niu et al., "mmWave communications for 5G: implementation challenges and advances," Science China Information Sciences, vol. 61, 2018. 


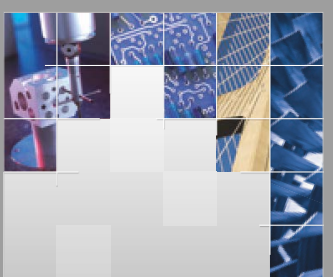

\section{Enfincering}
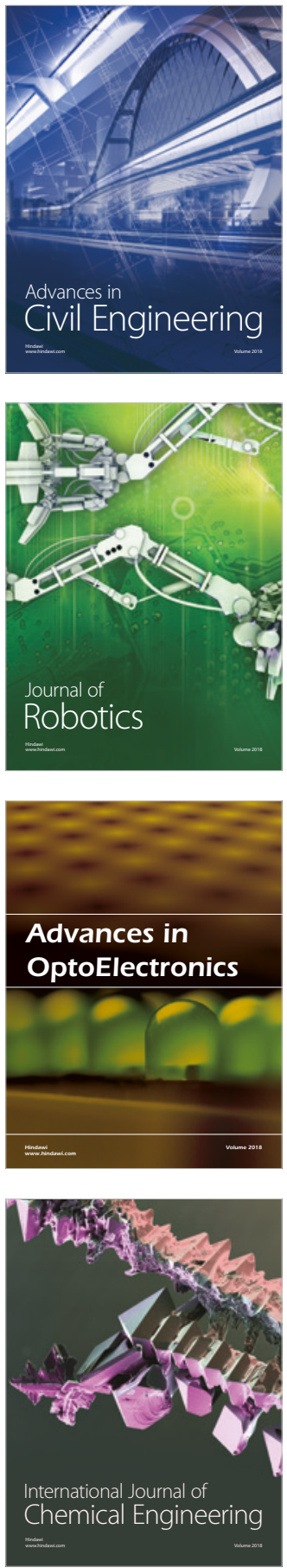

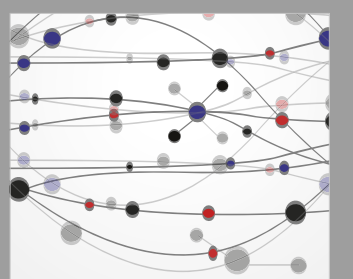

\section{Rotating \\ Machinery}

The Scientific World Journal

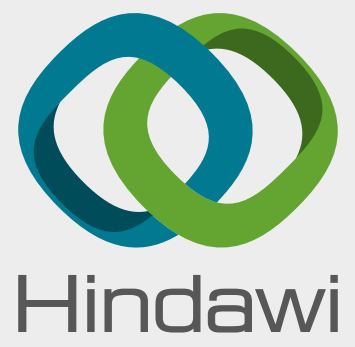

Submit your manuscripts at

www.hindawi.com
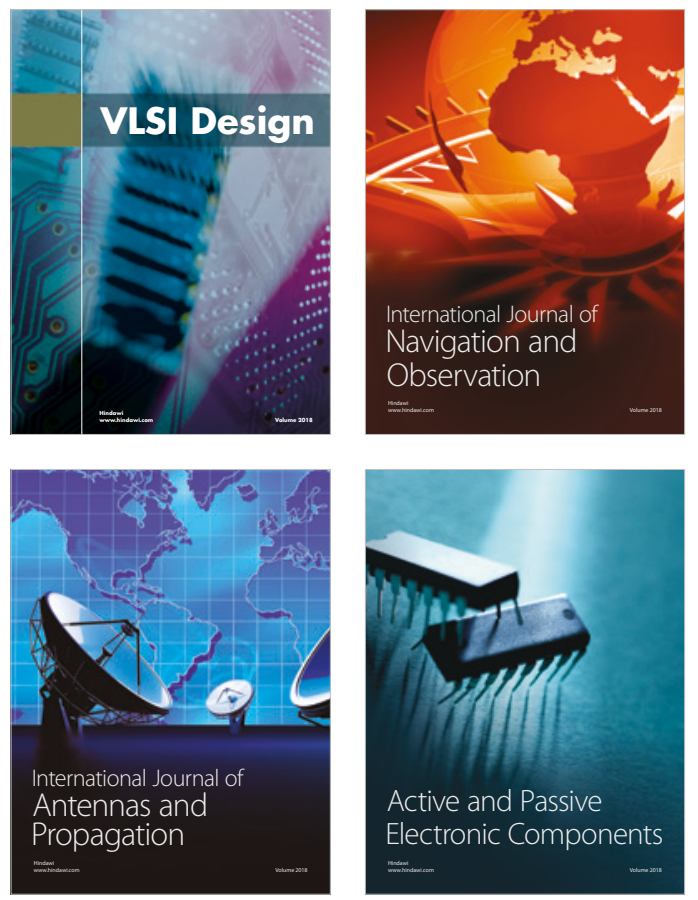
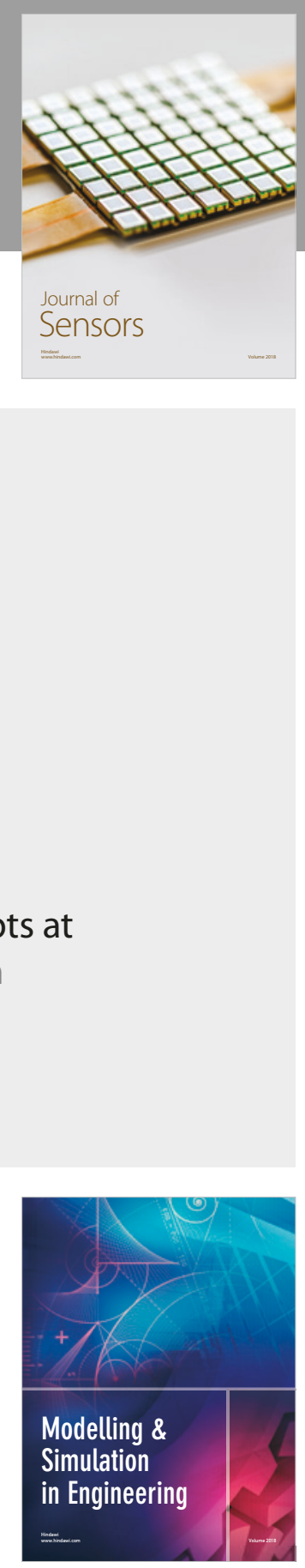

\section{Advances \\ Multimedia}
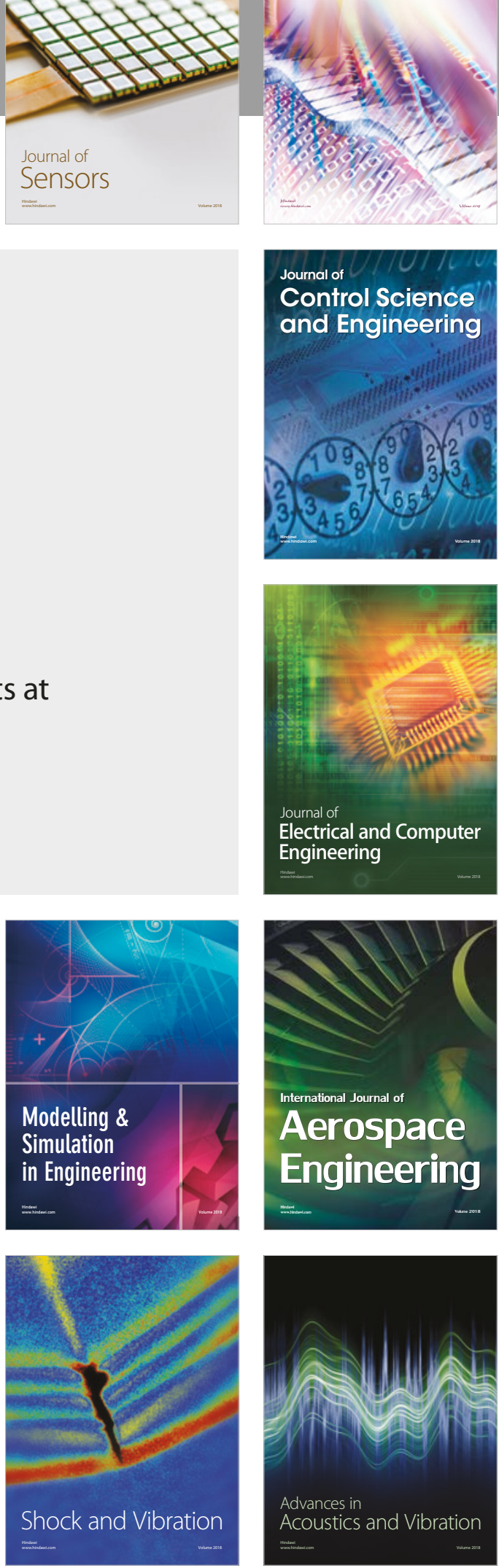